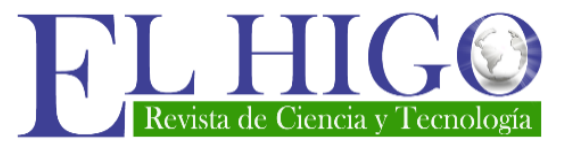

Vol. 06. pp. 30-35/Diciembre 2016
ISSN -P: 2413-192X ISSN-E: 2413-1911 Universidad Nacional de Ingeniería Sede Regional Norte

\title{
PROPUESTA DE REGLAMENTO TÉCNICO ORGANIZATIVO DE HIGIENE Y SEGURIDAD LABORAL Y MAPA DE RIESGOS DE LA EMPRESA CECOOPSEMEIN, SÉBACO, MATAGALPA, 2015.
}

\section{PROPOSAL OF THE ORGANIZATIONAL TECHNICAL REGULATION OF HYGIENE AND LABOR SAFETY AND MAP OF RISKS OF THE COMPANY CECOOPSEMEIN, SÉBACO, MATAGALPA, 2015}

\author{
Dominga Dalila González Cantarero ${ }^{8}$ \\ Erika Guadalupe Meza Pérez ${ }^{9}$ \\ Greyling Sarahí Aguirre Herrera ${ }^{10}$
}

RESUMEN: Con el objeto de garantizar las condiciones de seguridad e higiene ocupacional en la empresa CECOOPSEMEIN se realizaron encuestas a 37 trabajadores de la empresa incluyendo al personal directivo así como la utilización de técnicas de observación para conocer la situación actual de la empresa. Se logró identificar que en su mayoría, los trabajadores desconocen aspectos relacionados a este ámbito, lo cual muchas veces se pone de manifiesto en la incorrecta ejecución de las labores sin utilizar los EPP que la empresa proporciona. Se encontró que dentro de la empresa existen distintos factores de riesgo donde se destacan aquellos causados por agentes mecánicos, físicos, químicos, biológicos y ergonómicos, de los cuáles la probabilidad de ocurrencia de la mayoría de estos riesgos está entre media y alta. A partir de estos resultados se elaboró un reglamento técnico organizativo donde se plantean una serie de medidas preventivas y procedimientos que deben de seguir los colaboradores para garantizar la salud física y mental de cada uno de los trabajadores.

PALABRAS CLAVE: Factor; Probabilidad; Consecuencia; Enfermedad profesional.

ABSTRACT: In order to guarantee the safety and occupational hygiene conditions in the company CECOOPSEMEIN, surveys were carried out to 37 company employees, including the management staff, as well as the use of observation techniques to know the current situation of the company. It was possible to identify that the majority of workers are unaware of aspects related to this field, which is often evident in the incorrect execution of work without using the PPE that the company provides. It was found that within the company there are different risk factors which include those caused by mechanical, physical, chemical, biological and ergonomic agents, of which the probability of occurrence of most of these risks is between medium and high. Based on these results, a technical organizational regulation was drawn up, which sets out

\footnotetext{
${ }^{8}$ Docente UNI Sede Regional Norte, dalicanta@gmail.com

${ }^{9}$ Responsable de área de Formulación y ejecución de Proyectos, AURAMI SA, mezaerika919@gmail.com

${ }^{10}$ Gestora de seguros patrimoniales Seguros América S.A, gaguirre@segurosamerica.com.ni
} 
a series of preventive measures and procedures that employees must follow to ensure the physical and mental health of each worker.

KEYWORDS: Factor; Probability; Consequence; Professional illness.

\section{INTRODUCCIÓN}

Esta investigación contiene la propuesta de un Reglamento técnico organizativo de Higiene y Seguridad laboral y un mapa de riesgos para la empresa CECOOPSEMEIN, utilizando como herramienta principal el procedimiento metodológico para elaborar el Reglamento Técnico Organizativo RTO estipulado en la ley 618 artículo 63 de Nicaragua y el procedimiento técnico de higiene y seguridad del trabajo para la evaluación de riesgos en los centros de trabajo. De este modo marcará una base organizativa para que la empresa maneje lo que respecte a seguridad e higiene proyectadas en una serie de sugerencias preestablecidas cuyo beneficio será la adaptación de medidas preventivas que evitarán o reducirán los riesgos de accidentalidad en el trabajo y la conservación del buen estado físico y emocional de cada uno de los miembros que integra al factor humano de esta empresa nacional.

La empresa no contaba un RTO, ni un mapa de riesgos, lo que representa un factor importante que impide conocer la situación a la que está expuesta la empresa y limita la segura ejecución de las diferentes actividades que se desempeñan, aumentando así la incidencia de accidentalidad laboral y enfermedades profesionales por ende el incumplimiento de requisitos establecidos en la Ley No. 618. Ley General de Higiene y Seguridad del Trabajo.

Se planteó una propuesta de un RTO y un mapa de riesgos dando respuesta a la necesidad en el cual se establecen los parámetros sobre medidas que la empresa debe asumir para disminuir la incidencia de accidentes a fin de generar un ambiente laboral seguro, higiénico y productivo, estableciendo las obligaciones que poseen tanto los empleadores como los trabajadores.

\section{METODOLOGÍA}

La investigación se planteó desde un enfoque explicativo y descriptivo, debido a que la investigación explicativa está diseñada para obtener un análisis preliminar de situaciones para posteriormente exponer definiciones o suposiciones ampliamente relacionados con el tema de estudio, esta es apropiada en las etapas iniciales del proceso investigativo debido a que se desea captar un análisis de la situación actual de la cooperativa y a través de la investigación descriptiva se analizaron y midieron diferentes aspectos relacionados con el factor humano, opiniones de las personas a través de encuestas realizadas a los mismos para evaluar una serie de aspectos orientados a ser descritos a medida que se van investigando.

El universo y muestra de esta investigación son todos los colaboradores con los que contaba la empresa en su totalidad, siendo estos una cantidad de 82 trabajadores.

La metodología empleada consistió en visitas periódicas, donde se aplicaron los métodos y técnicas de observación, entrevistas a encargados de las líneas de producción así como encuesta al presidente de dicha cooperativa, las cuales fueron aplicadas a un total de treinta y 
siete personas. Todo ello con el fin de recopilar toda la información necesaria de la situación actual de la empresa para la evaluación de riesgos y elaboración del RTO a través de la metodología establecida por el MITRAB

\section{RESULTADOS Y DISCUSIÓN}

Dentro de la empresa, se realizan una serie de actividades donde los operarios participan directamente de ellos y hacen posible la funcionalidad de los mismos. Existen diversos factores que pueden influir en el correcto desempeño de las actividades como la falta de señalizaciones dentro de las instalaciones, riesgos físicos, ergonómicos, biológicos y químicos, los cuales podrían afectar la salud e integridad física de las personas que laboran e incluso podrían salir afectados los visitantes y clientes (figura 1 y 2 ).

Figura 1: Maquinaria utilizada

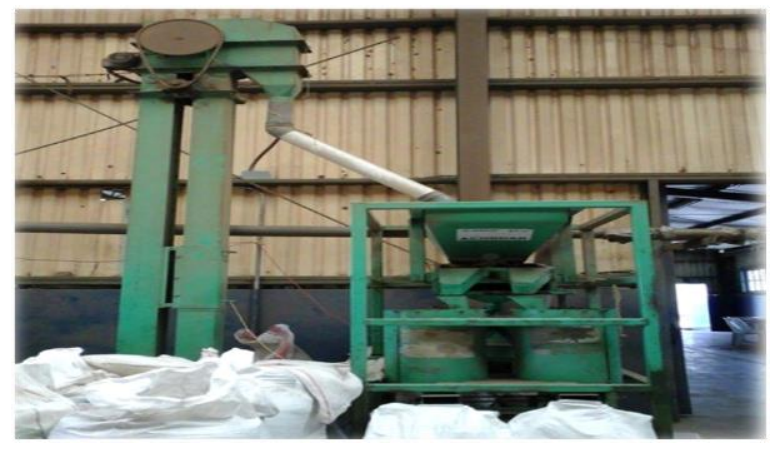

Fuente: Propia
Figura 2: Escogido manual

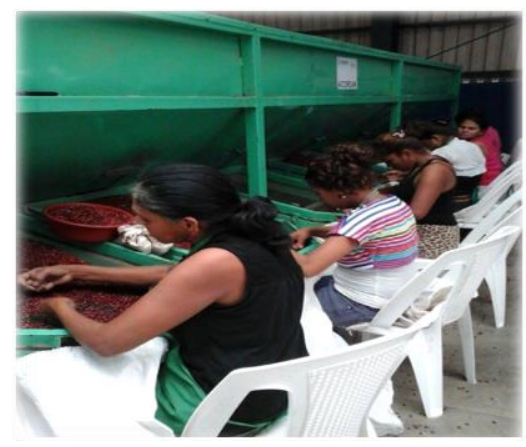

Fuente: Propia

Primeramente se describieron los procesos productivos de sus productos principales (frijol rojo seda y chía), posteriormente se detallaron cada uno de los puestos existentes en la empresa destacando cada una de sus funciones. Se identificaron los factores de riesgos a los que estaban expuestos los trabajadores por puesto de trabajo mediante la observación directa y visitas periódicas a las instalaciones y el proceso productivo de la empresa, encuestas a los operarios y entrevistas a los jefes de planta. Se determinaron los riesgos que pudieran provocar alguna lesión o enfermedades profesionales a los trabajadores o daños materiales, tomando como base la fuente del peligro, el tipo de daño que ocasionar y los efectos que genera a los trabajadores a la hora de efectuar sus funciones.

A través de la estimación, valoración y caracterización de las probabilidades de ocurrencia de peligros se apreció que la mayoría de éstos, según el Acuerdo ministerial JCHG-000-08-09 oscila entre media y alta, donde los riesgos de enfermedades y accidentes profesionales de mayor incidencia son aquellos generados a partir de una mala organización de equipos $u$ organización en el área de almacén, entre los que predominaban los riesgos ergonómicos que podrían desencadenar accidentes y enfermedades ocasionadas por agentes microbiológicos, musculo esqueléticos y riesgos mecánicos que de manera directa afectarían la integridad física de los trabajadores, a parte de la mala organización que existe también está inmersa las prácticas inadecuadas de trabajo. Todo ello debido a que las capacitaciones que se brindan no se hacen de manera periódica, ya que se constató que un $63 \%$ de los encuestados desconocen 
aspectos asociados a higiene y seguridad del trabajo, de hecho esto se pudo comprobar con la observación de la ejecución de sus labores.

Luego de ello se realizó el mapa de riesgos donde se representó de forma gráfica el diseño de las instalaciones de la empresa con los riesgos identificados indicando el tipo y estimación de riesgo con su color representativo, el número de trabajadores expuestos de acuerdo a lo establecido en el acuerdo ministerial de evaluación de riesgos. Se elaboró el RTO siguiendo la metodología para su elaboración, el cual contempla el objetivo del mismo, ámbito de aplicación, las obligaciones tanto de los trabajadores como del empleador, la elaboración de la matriz de riesgos donde se contemplaban cada factores con su estimación así como medidas preventivas y procedimientos de trabajo para cada riesgo encontrado, las sanciones que podría conllevar el desacato de este reglamento, entre otros aspectos. Así mismo, se realizó una capacitación en la parte de higiene y seguridad laboral y las 5'S para informar a los colaboradores los factores de riesgos a los que estaban expuestos y la importancia del uso de EPP y el orden en el área de trabajo como medidas preventivas (figura 3 y 4 ).

Figura 1: Capacitación HyST y 5’S
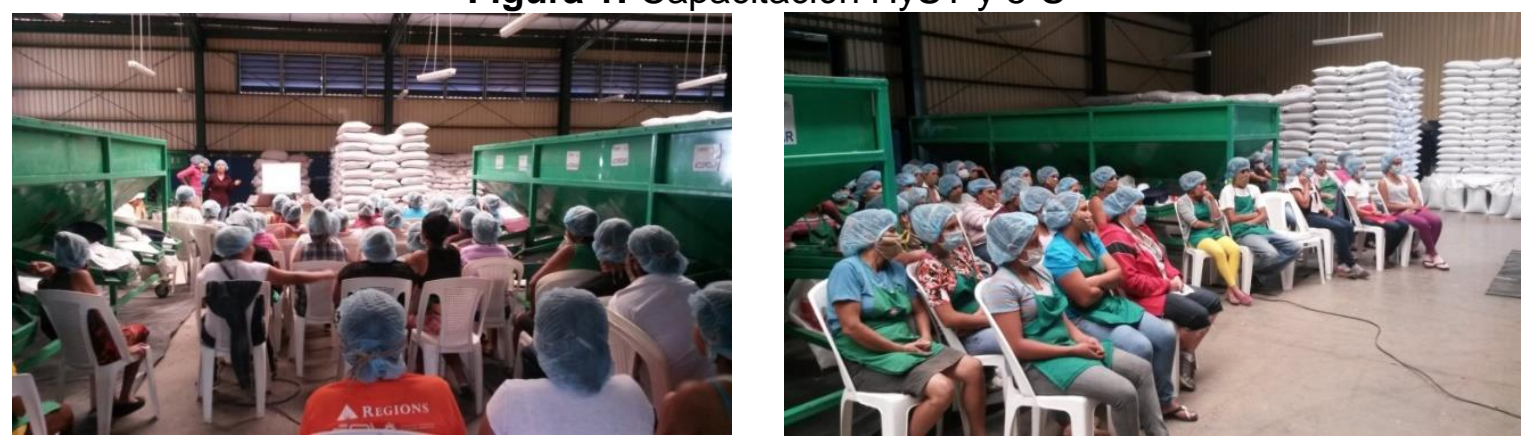

Fuente: Propia

\section{CONCLUSIONES}

A través de las visitas periódicas e interacción con los trabajadores y con el medio en el que se desarrolla la actividad económica de CECOOPSEMEIN R.L se conoció la verdadera situación de las condiciones de higiene y seguridad dentro de ésta empresa.

No existía un departamento de seguridad e higiene laboral, pero sí contaban con una licencia de operación; No se tenía conformada una comisión mixta; No existe un reglamento técnico organizativo; No se llevaba un registro del índice de accidentalidad de los trabajadores; No existía un mapa de riesgos, los EPP se brindaban de manera gratuita tanto a operarios como a visitantes.

A partir de los hallazgos se dedujo que la empresa debe instruir al personal a través de capacitaciones periódicas en materia de seguridad e higiene laboral, donde se refleje los riesgos y peligros a los cuales están expuestos; Evaluar e inspeccionar periódicamente las instalaciones en general, el equipo de trabajo y las actividades de los trabajadores con el fin de detectar condiciones de riesgo. 
Se debe formar una comisión mixta la cual velará por el cumplimiento de valoración de riesgos y condiciones de trabajo actual para poder aplicar las mejoras pertinentes dentro de la empresa; Colocar el mapa de riesgos en un lugar visible para los visitantes, clientes y personal en general tal y como se indica el art 18, numero 4 y 5 de Ley 618, así como la señalización que indique las rutas de evacuación, riesgo eléctrico, salidas de emergencia, exigencias e instrucciones del uso de equipos e indumentaria de protección personal y delimitación de las áreas correspondiente en colores visibles y en una posición adecuada.

Mantener el orden, aseo y limpieza dentro, fuera y alrededores de la empresa; Registrar el índice de accidentalidad y enfermedades profesionales. Es importante dar seguimiento a los riesgos encontrados, cambios efectuados y charlas brindadas, todo esto para mantener controlado el avance que se ha obtenido y así mismo obtener mayores resultados; Garantizar los EPP de acuerdo al puesto y el tipo de riesgo al que este expuesto el trabajador, siendo de uso obligatorio tanto para trabajadores como para visitantes; Cumplir con todas las normas preestablecidas.

\section{ABREVIATURAS}

RTO: Reglamento Técnico Organizativo.

CECOOPSEMEIN: Central de Cooperativas de Servicios Múltiples Exportaciones e Importaciones del Norte.

MITRAB: Ministerio del Trabajo.

EPP: Equipos de Protección Personal.

\section{REFERENCIAS}

Asamblea General de la República. (13 de julio de 2007). ley 618. Obtenido de www.mitrab.gob.ni/documentos/bibliotecavirtual/COMPILACION\%20de\%20ley\%20y\%20normati vas\%20en\%20materia\%20de\%20higiene.pdf/at download/file

Asamblea Nacional . (s.f.). Procedimiento Técnico de Higiene y seguridad del trabajo para la evaluación de riesgos en los centros de trabajo. Nicaragua.

Seguridad y Salud en el trabajo. (2015). Los mapas de riesgos. Obtenido de http://normaohsas18001.blogspot.com/2013/01/los-mapas-deriesgos.html 

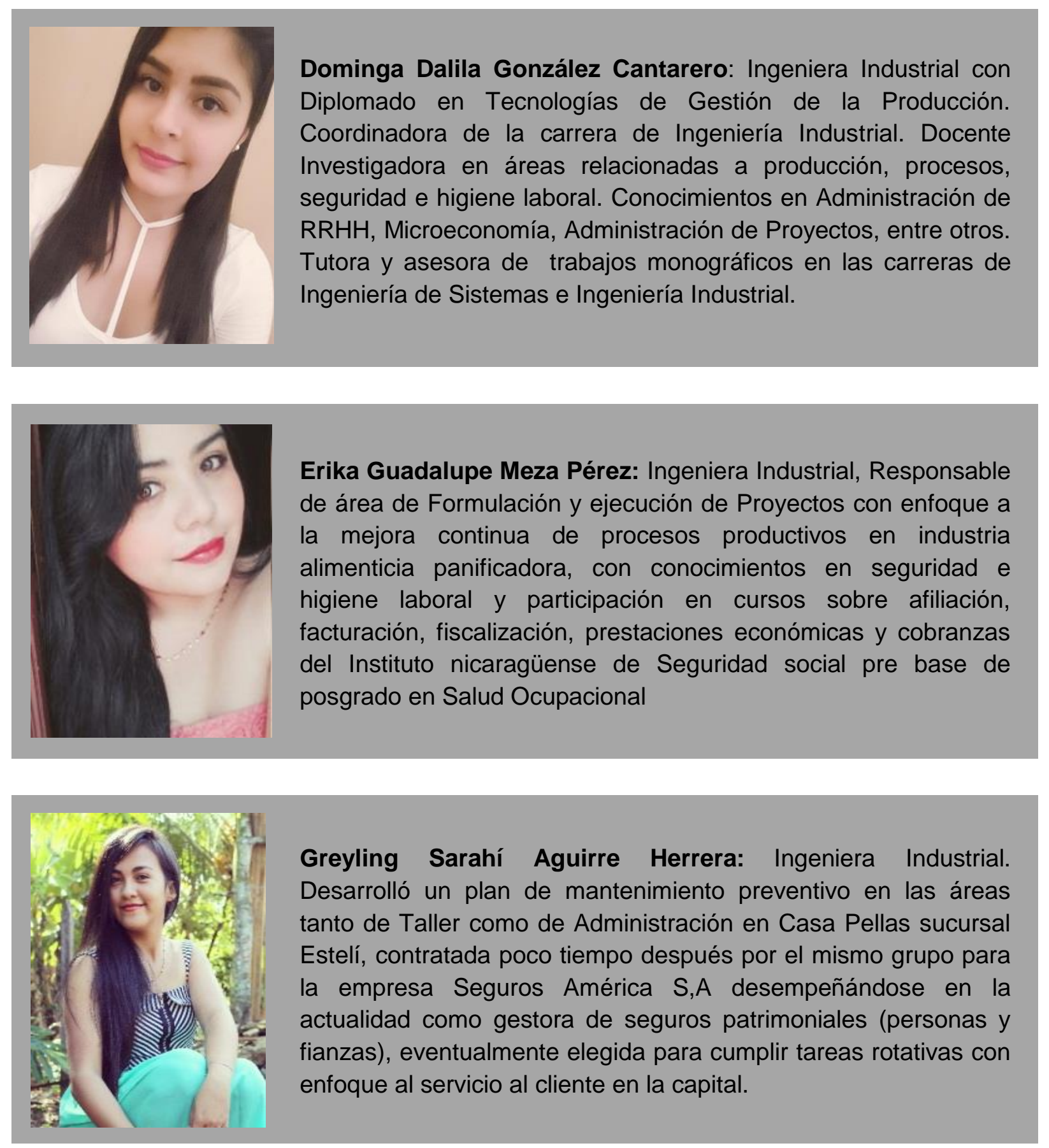